\title{
Development of Diagnostic Classifier for Ultrasound Liver Lesion Images
}

\author{
V. Ulagamuthalvi \\ Research Scholar \\ Sathayabama University, Chennai-119,INDIA
}

\author{
D. Sridharan \\ Associate Professor \\ Anna University, Chennai 600025, ,INDIA
}

\begin{abstract}
Liver cancer is the fifth most common cancer worldwide in men and eighth in women, and is one of the few cancers still on the rise. In this study, we focus on Development of Diagnostic Classifier for Ultrasound liver lesion. Naturaly, Ultrasound liver lesion images are having more spackle noise. Developing classifier for ultrasound liver lesion image is a challenging task. We approach fully automatic machine learning system for developing this classifier. First, we segment the liver image by calculating the textural features from co-occurrence matrix and run length method. For classification, Support Vector Machine is used based on the risk bounds of statistical learning theory. The textural features for different features methods are given as input to the SVM individually. Performance analysis train and test datasets carried out separately using SVM Model. Whenever an ultrasonic liver lesion image is given to the SVM classifier system, the features are calculated, classified, as normal, benign and malignant liver lesion. We hope the result will be helpful to the physician to identify the liver cancer in non invasive method.
\end{abstract}

Key Words: Segmentation, Support Vector Machine, Ultrasound Liver Lesion, Co-occurance Matrix

\section{INTRODUCTION}

Classifications of ultrasound liver lesion images are very difficult task in the image processing. In the medical field computer are now being used virtually in every aspect of modern medicine. Computers are used widely in medical research, where there is a vital need for better microelectronic sensors for data acquisition. Diagnosis by ultrasound imaging is a cost effective approach to ascertain the disease in earlier stage. Liver diseases are considered seriously because of the liver's vital importance to human beings. There are two classes of liver tumors: benign and malignant [8]. Ultrasound image is a powerful tool for characterizing the state of soft tissues for medical diagnostic purposes. Ultrasound has been extremely valuable in differentiating a simple liver lesion from other liver masses. An approach has been made in this research to design a diagnostic classifier system for liver lesion in ultrasound images using image texture features in non-invasive manner.

Image processing modifies pictures to improve them (enhancement, restoration), extract information (analysis, recognition), and change their structure (composition, image editing). Images can be processed by optical, photographic, and electronic means, but image processing using digital computers is the most common method because digital methods are fast, flexible, and precise[14].

So, the proposed system we applied the co-occurrence matrix features and gray level run-length features for identifying the seed point for given ultrasound liver images. After the detection of automated seed point we have to segment the liver image applying the region growing algorithm using gray space map and
Otsu algorithm. After segmentation of the image we analyzed and calculated texture features parameters to classified, as normal, benign and malignant liver lesion. We explains the image preprocessing procedures, segmentation of image in the section 2, Computation of different image texture features of different feature extraction methods and machine learning classifier SVM used for classification of Ultrasound Liver lesion image using texture in section 3 Trialing result in section 4 and conclusion in the section 5 .

\section{MATERIAL AND METHODOLOGY 2.1. Image Preprocessing, Filtering and Image Enhancement}

An Ultrasound liver lesion images has been taken for this study. The preprocessing step typically is used for reduce the noise and to prepare the ultrasound liver image for further processing such as segmentation and classification. To get a high-pass filter, the general procedure is to apply a low-pass filter to the original image and then subtract this low-frequency image from the original image. The result is then an image containing only high frequencies. Sometimes it is desired to enhance the high frequencies without removing the low frequencies. The preprocessing work could be done for removing the noise of the images. After the removal of noise from the image we applied the histogram to identify the maximum of the intensity value. Then we applied the techniques for segmentation of the ultrasound liver lesion.

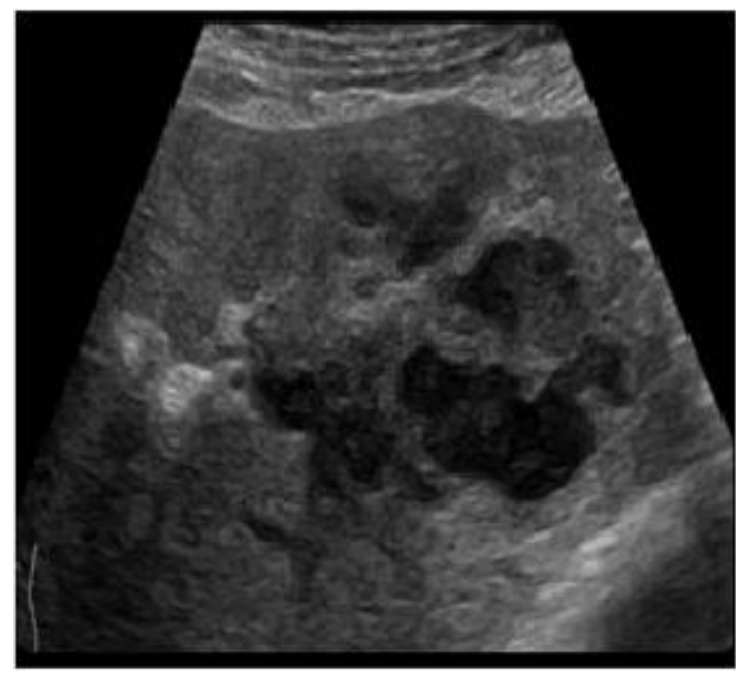

Fig 1. Original Image 


\subsection{ROI Selection and Segmentation}

Segmentation is played an important role in the image processing. Normally, Segmentation of Ultrasound images are very difficult because it contains more speckle noise. An automatic algorithm is proposed based on the region growing frame work. The algorithm select the initial seed automaticallyusing of coocurrance matrix and runlength parameters.. Based on the histogram, it is observed that they have gray values in the range (235-255). Algorithm of auromatic seed point selected region-growing algorithm.

1. Read the input image.

2. Scan the image fully.

3. Search the pixel fro the high intensityvalue that is seed.

4. Find the neighbor pixel using connectivity. If the neighbor has the value within the tolerance, add the pixel and grow the region.

5. Repeat the step 4 till a complete region of similar intensity odf connected region is obtained.

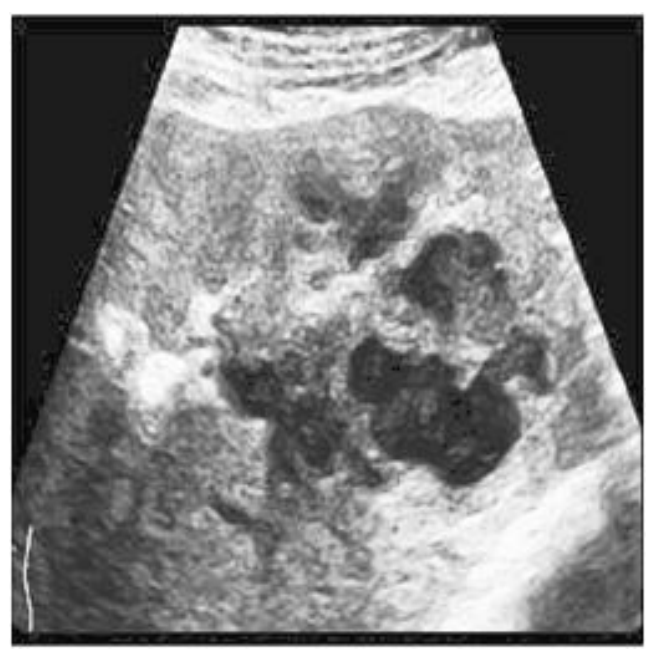

Fig 2. Histogram Equalized Image

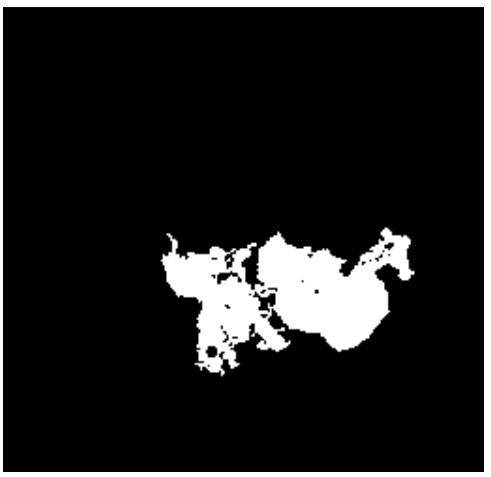

Fig 3. Segmented Image

\subsection{Gray Space Map}

The algorithm of region growing is very simple. We compute the seed gray level: $U$, then look for structures which have the same gray level than the seed overlapping the seed position. At the second iteration, we look for structures having a small gray level difference from the seed. In other words, we define a set of gray levels from U-D to U+D. Then we keep those structures which overlap the seed position. At each iteration we increase the difference $\mathrm{D}$ by 1 . In this way structures which are closed from a spatial AND intensity point of view to the seed are highlighted with higher values [5]. In new image if we far spatially and from an intensity the point of view from the seed, the lower intensity is labeled. The resulted image is Gray Space map of image.

\section{TEXTURE FEATURE EXTRACTION METHODS}

Texture feature extraction is the procedure of generating descriptions of a textured surface in terms of measurable parameters. The extracted features represent the relevant properties of the surface, and may be used with a classifier. The following textural features groups are used in the proposed system, First order statistics (Histogram), Second order statistics, Run - length matrices and Wavelet features.

\subsection{Texture Descriptors}

Texture descriptors based on the first order fray levels static [6]: Energy, Entrophy, and Mean

Texture descriptor based on the Gray Lavels Co-occurance Matrix (GLCM) - Second order gray levels statistics [7]: Homogenetity, Entrophy, Energy, Contrast, Varience and Correlation.[17].

In this method, the features are derived from the gray level histogram. The digital image can be represented as a twodimensional array in the computer. For the digital images, 8 bits are sufficient and the gray-level values range from 0 to 255 . Lower values are attributed to darker pixels, and higher values to brighter pixels. Therefore 0 represents the black and white represents the 255 .

A Co-Occurrence Matrix is square matrices of relative frequencies with which two neighboring pixels separated by distance $\mathrm{d}$ at orientation q occur in the image, one with gray level $i$ and the other with gray level $\mathrm{j}$ (Joaquim Jose Furtado $1 * 2010$ ). Therefore, a square matrix that has the size of the largest pixel value in the image and presents the relative frequency distributions of gray levels and describe how often one gray level will appear in a specified spatial. In our project, 2 textural features were calculated from the COM for direction $\mathrm{h}$ values of $0^{\circ}$ and a distance $\mathrm{d}$ of 1 . In this work the co-occurrence features energy and entropy which can easily differentiate nonhomogeneous region from homogeneous region are considered. It is a measure the homogeneousness of the image and can be calculated from the normalized COM. Energy is expected to be high if the occurrence of repeated pixel pairs is high. It denotes the normalized co-occurrence matrix by total number of the occurrence of two neighboring pixels between I gray-intensity at vertical direction and angle $\Theta$. Entropy gives a measure of complexity of the image. Complex textures tend to have higher entropy if the gray levels are distributed randomly through out of the image.

These two parameters can identify seed pixel from the abnormal region of the ultrasound liver cancer tumor images. Some times for some cases the normal liver region also can appear be a homogeneous. So to avoid that situation by calculating the run length features.

\subsection{SVM Classifier}

Support Vector Machines (SVM) based on tha concept of decision planes that defines decision boundaries. A plane is one that separates between a set of objects having different class members.This is a classic example of a linear classifier, which is a classifier that separates a set of object into their respective groups.Most classification tasks typically involve more than two classes. To reduce a problem to a set of two-class binary problem 
with a divide and conquar strategy is possible.In terms of classification the magnitude of the classifier's output is unimportant, so it suffices to say that an input vector shall be binary linear decision boundaries placed into the +1 class whenth boundary is greater than the 0 and -1 class when the boundary reaches lessthan 0 . The decision boundary that splits the two classes.

A kernel can be considered as a data transformation. Many different choices for the kernel namely polynomial, Radial Basis Indicator Function (RBF) and neural network indicator function are possible. SVMs do not suffer overfitting to the same extent as neural networks, as only the training data vectors that are needed to maximise the separation of the classes are used to define the decision boundary. These vectors are termed the support vectors. Testing data is extracted from each preprocessed, segmented image, and stored in a text file without class labels. The testing dataset is given as the input to the SVM Model for classification.

\section{RESULTS}

We describe the performance of the SVM classifier to identification of liver lesion from different textural features method used for segmentation and classification. The texture features are extracted from the high intensity value using Otsu's thresholding method. SVM used as a classifier. This classifier offers the better results in identifying the malignant liver tumor from the normal. In some cases the benign tumors are misclassified as a normal liver. The intensity value of the normal liver and begin may be same. The overall accuracy of classification value $96.72 \%$ approximately for the ultrasound liver images.

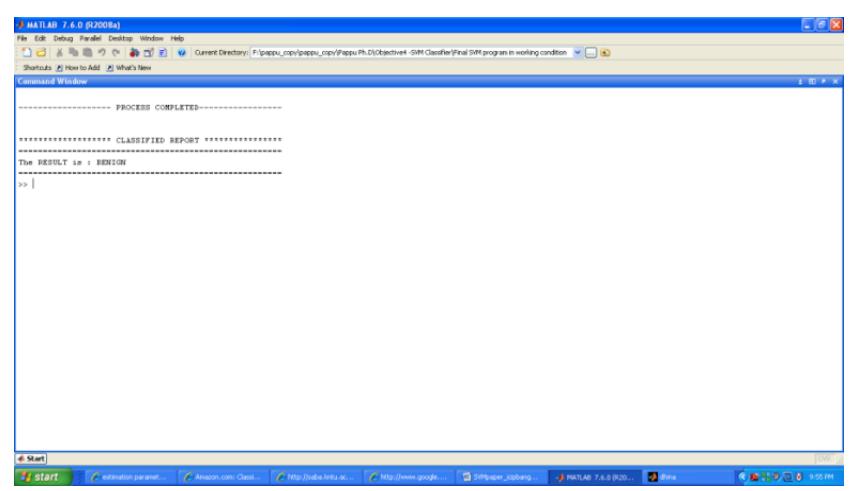

Fig. 4. Shows the diagnostic classification in the MATLAB command window.

\section{CONCLUSION}

This paper proposes the Development of Diagnostic Classifier for Ultrasound Liver Lesion Images. First, we detect the seed point for the given ultrasound liver image automatically using features of co-occurrence matrix and run length method. Second, we segment the ultrasound liver images using of gray space map and Otsu method. Finally using SVM classifier we classified the ultrasound liver lesion image as normal, benign and malignant. We hope this system will help the physician to diagnose the liver lesion with non invasive method.

\section{ACKNOWLEDGEMENT}

The authors convey their heartfelt thanks to Dr. G. Kulanthaivel, Associate Professor, National Institute of Technical Teachers Training and Research , Chennai,India for his magnanimous help and spending their valuable time in giving suggestions at different stages of the work.

\section{REFERENCES}

[1] Asmita, A. Moghe, Jyoti Singhai \& S.C Shrivastava(2011) :Automatic Threshold based Liver Lesion Segmentation in Abdominal 2D-CT Images International Journal of Image Processing (IJIP), $5: 2:$ pp166-176.

[2] Haruka, D.and Teruak, A. (2007): Characterization of spatiotemporal stress distribution during food fracture by image texture analysis Methods:Journal of Food Engineering : 81: pp.429-436.

[3] Joaquim Jose Furtado1*(2010) , Zhihua Cai1 \& Liu Xiaobo1,: Digital Image Processing: Supervised Classification using Genetic Algorithm in MATLAB Toolbox" Report and Opinion;2(6) .

[4] Koss JE, Newman FD, Johnson TK, Krich DL.(1999) Abdominal organ segmentation using texture transform and Hopfield neural network. IEEE Trans Med Imaging;18:640

[5] Kulanthaivel G. and Ravindran G.( 2003)"Web Based Diagnostic aid for Kidney Lesions By Image Texture Parameters", Biennial Conference of Indian Association for Medical Informatics, Chandigarh,p.14.

[6] R C GonZalez, R .E. Woods (2001) - Digital Image Processing, Second Edition. Prentice-Hall.

[7] A.Materkaand M.Stezeleceki,(1998) "Textural Analysis methods-A Review “, COST BII Report ,Brussels, Technical University of Lonz, Institute of Elecronics ul. Stefanowskiego -18,90-124.

[8] Kumar ,S.S. , Dr Moni R.S.(2010) : Diagnosis of Liver Tumor from CT Images Using Fast Discrete Curvelet Transform", IJCA Special Issue on "Computer Aided Soft Computing Techniques for Imaging and Biomedical Applications" CASCT, Pp1-6.

[9] Mancas M., B. Gosselin And B. Macq,(2005) "Segmentation Using a Region Growing Thresholding" 4th Image processing : algorithms and systems :5: pp. 388-398.

[10] Miller P, Astley S. (1992): Classification of breast tissue by texture analysis. Image Vision Computer;10:277-282.

[11] Nobel A.J and Boukerroui D.:Ultrasound Image Segmentation: A survey: IEEE Trans On Medical Imaging :25:.8:pp.987-1010.

[12] Otsu, N. (1979) :A Threshold Selection Method from GrayLevel Histogram: IEEE Trans. Systems Man, and Cybernetics: 9: pp. 62-66,.

[13] Padma,.A,.Sukenesh.R ( 2011) , :Automatic Classification and Segmentation of Brain Tumor in CT images using optimal Gray Level Run lengthTexture Features", IJACSA) International Journal of Advanced Computer Science and Applications: Vol. 2, No. 10:pp53-59.

[14] Withey, D.J. and Koles, Z.J. (2007) "Three Generations of Medical Image Segmentation: Methods and Available Software,"International Journal of Bioelectromagnetism : $9: 2:$ pp 67-68.

[15]Wu CM, Chen YC. (1992):Texture features for classification of ultrasonic liver images. IEEE Trans Med Imaging;11:141-52.

[16]Xiao, G. Brady, .M Noble J. A. and Zhang, Y. (2002.):Segmentation of ultrasound B-mode images with 
intensity in homogeneity correction: IEEE Trans. On Med. Imaging : 21,:1: pp. 48-57.

[17]Horia Stefaneseu.Radu Badea.Monica Lupsor.Simona Tripon.Teodora pop,(2007)"Telemedicine Network for Ultrasound Screening of HCC" ,Ist International Conf. on Advancements of Medicine and Health Care Through Technology (MediTech2007), pp. 107-110.
[18] Xie J, Jiang Y, Tsui HT.( 2005) : Segmentation of kidney from ultrasound images based on texture and shape priors. IEEE Trans Med Imaging:;24:45-57.

[19] V.Ulagamuthalvi,D.Sridharan,(2012)"Automatic Identification of Ultrasound Liver Cancer Tumor Using Support Vector Machine" Proceeding of International Conf. on Emerging Trends in Computer and Electronics Engg(ICETCEE2012), pp : 41-43. 